\title{
GAIA Major Congenital Anomalies Level of Diagnostic Certainty
}

National Cancer Institute

\section{Source}

National Cancer Institute. GAIA Major Congenital Anomalies Level of Diagnostic

Certainty. NCI Thesaurus. Code C126842.

A classification of maternal and fetal outcomes relating to major congenital anomalies, developed by the Global Alignment of Immunization safety Assessment in pregnancy, based on the extent to which the diagnosis has been confirmed. 